\title{
GBP1 promotes erlotinib resistance via PGK1-activated EMT signaling in non-small cell lung cancer
}

\author{
LIFANG CHENG $^{1,2}$, LANYING GOU ${ }^{1}$, TING WEI ${ }^{1}$ and JIAN ZHANG $^{1}$ \\ ${ }^{1}$ Department of Oncology, Zhujiang Hospital, Southern Medical University, Guangzhou, Guangdong 510282; \\ ${ }^{2}$ Department of Oncology, Shenzhen Samii International Medical Center, Shenzhen, Guangdong 518118, P.R. China
}

Received March 5, 2020; Accepted June 10, 2020

DOI: $10.3892 /$ ijo.2020.5086

\begin{abstract}
Erlotinib, an epidermal growth factor receptor tyrosine kinase inhibitor (EGFR-TKI), is widely applied as a first-line treatment for non-small cell lung cancer (NSCLC) and greatly improves the clinical outcomes of patients. However, acquired resistance to EGFR-TKIs remains a major clinical challenge. Here, we identified guanylate-binding protein-1 (GBP1) as a novel protein related to erlotinib resistance, and explored the specific mechanism by which GBP1 is involved in erlotinib resistance. First, the human NSCLC cells PC9ER and HCC827ER were generated by exposing cells to increasing concentrations of erlotinib over 6 months. We screened different genes between erlotinib-sensitive and erlotinib-resistant cells with data from the Gene Expression Omnibus database and detected the expression of these genes in erlotinib-resistant and erlotinib-sensitive cells by quantitative real-time polymerase chain reaction (qPCR). Moreover, we constructed GBP1-knockdown and GBP1-overexpressing cells to determine the $\mathrm{IC}_{50}$ value of erlotinib, to perform an apoptosis assay and to examine cell cycle distribution. A subcutaneous tumorigenesis test was used to analyze how GBP1 affects erlotinib resistance. Then, mass spectrometry analysis and coimmunoprecipitation were performed to verify the interaction between GBP1 and phosphoglycerate kinase 1 (PGK1). Changes in epithelial-mesenchymal transition (EMT)-related markers were observed following the upregulation and downregulation of PGK1 expression. Finally, a rescue experiment was used to determine whether GBP1 regulates EMT through PGK1. In the present study, GBP1 was significantly upregulated in erlotinib-resistant cells, compared with erlotinib-sensitive
\end{abstract}

Correspondence to: Professor Jian Zhang or Dr Ting Wei, Department of Oncology, Zhujiang Hospital, Southern Medical University, 253 Industrial Avenue, Guangzhou, Guangdong 510282, P.R. China

E-mail: blacktiger@139.com

E-mail: 258077534@qq.com

Key words: guanylate-binding protein-1, erlotinib resistance, non-small cell lung cancer, phosphoglycerate kinase 1, epithelial-mesenchymal transition cells. In vitro and in vivo experiments showed that upregulated GBP1 expression contributed to erlotinib resistance, while decreased GBP1 expression had the opposite effect. As shown by performing survival analysis, high GBP1 expression predicted poor prognosis in patients with lung adenocarcinoma. Furthermore, the interaction between GBP1 and PGK1 was confirmed, and a rescue experiment revealed that GBP1 regulates EMT via PGK1. Finally, functional experiments showed that EMT is involved in erlotinib resistance. Our study suggests that GBP1 regulates erlotinib resistance via PGK1-mediated EMT signaling, suggesting GBP1 as a potential therapeutic target in erlotinib-resistant NSCLC.

\section{Introduction}

Non-small cell lung cancer (NSCLC) is the most common histological subtype of lung cancer, accounting for approximately $80 \%$ of all lung cancer (1). Erlotinib remarkably prolongs the survival time of NSCLC patients with epidermal growth factor receptor (EGFR) mutations, yet following 8-16 months, treatment is often accompanied by drug resistance and disease progression (2). To date, several resistance mechanisms have been explored, including the secondary T790M mutation in EGFR, small cell transition, MET or human epidermal growth factor receptor 2 (HER2) amplification (3), and epithelial-mesenchymal transition (EMT) (4). However, as these events do not adequately elucidate for all NSCLC cases (5), the strategies to overcome erlotinib resistance deserve further study.

Guanylate-binding protein-1 (GBP1) is a guanosine-5'-triphosphate-binding protein in the dynamin superfamily, and regulates multiple cell functions (6). A report showed that GBP1 modulated the migration and invasion of oral cavity squamous carcinoma cells in vitro (7). Yamakita et al, discovered that GBP1 promoted lung adenocarcinoma invasiveness (8). GBP1 was also reported to be related to paclitaxel resistance in ovarian cancer cell lines (9). Therefore GBP1 is associated with tumor progression and chemotherapy drug resistance. However, the relationship between GBP1 and epidermal growth factor receptor tyrosine kinase inhibitor (EGFR-TKI) resistance is unclear. In the present study, we found that GBP1 was expressed at higher levels in resistant cells than in sensitive cells, thus we further aimed to ascertain whether GBP1 plays a role in erlotinib resistance. 
To investigate the role of GBP1, we used a small hairpin RNA (shRNA) targeting GBP1 to knock down GBP1 expression in erlotinib-resistant cells. Cell Counting Kit-8 (CCK-8) experiment showed that erlotinib-resistant cells were more sensitive to erlotinib after GBP1 knockdown. Then, we overexpressed GBP1 in erlotinib-sensitive cells by transfected with an overexpression plasmid and found that upregulation of GBP1 contributed to a higher erlotinib $\mathrm{IC}_{50}$ than in control cells. Our data showed that GBP1 overexpression inhibited apoptosis and caused a G1 to $S$ phase transition, and the opposite effects were observed in GBP1-knockdown cells. In addition, we confirmed that GBP1 knockdown in resistant cells markedly reduced the tumor volume in vivo. In addition, high GBP1 expression predicted poor prognosis in a survival analysis of clinical samples. The above results indicated that GBP1 promotes erlotinib resistance.

To further study the mechanism by which GBP1 regulates EGFR-TKI resistance, we performed mass spectrometry analysis of GBP1 in cells. After screening by mass spectrometry, we identified PGK1 as a GBP1-interacting protein. PGK1, an important metabolic enzyme, is overexpressed in many cancers, such as glioblastoma (10) and triple-negative breast cancer (11). Moreover, PGK1 promotes the migration and invasion of NSCLC cells via the AKT/mTOR signaling pathway (12). Here, we found that PGK1 is involved in erlotinib resistance in NSCLC by activating the EMT pathway, causing the loss of epithelial markers and the gain of mesenchymal markers in cancer cells. Subsequently, our results showed that GBP1 modulates erlotinib resistance through EMT mediated by PGK1.

In summary we found that GBP1 caused resistance to erlotinib. The knockdown of GBP1 expression significantly sensitized NSCLC cells to erlotinib in vitro and in vivo. Our discoveries provide support for the feasibility of targeting GBP1 to overcome erlotinib resistance.

\section{Materials and methods}

Differential gene selection. Firstly, we selected 3 datasets concerning human NSCLC samples from the Gene Expression Omnibus (GEO) database (http://www.ncbi. nlm.nih.gov/geo/), in which GSE64322 (http://www.ncbi. nlm.nih.gov/geo/qyery/acc.cgi?acc $=$ GSE64322) showed molecular changes between EGFR-TKI-sensitive and EGFR-TKI-resistant NSCLCs by transforming to NSCLC cells, GSE38310 (http://www.ncbi.nlm.nih.gov/geo/ qyery/acc.cgi?acc=GSE38310) (14) displays gene expression differences between HCC827 and HCC827ER cells and GSE34228 (http://www.ncbi.nlm.nih.gov/geo/qyery/acc. cgi?acc $=$ GSE34228) (15) contains genome expression differences of PC9 and PC9ER cells. Then, the R limma package (16) was used to determine which genes were different in the various gene sets. The results indicated that $G B P 1, T G M 2$, PAPPA, HAS3, CCD2A, HMGA2, INSL3, LDB3, PMIL and $S L C 35 B 3$ were significantly differentially expressed between EGFR-TKI-resistant and EGFR-TKI-sensitive lung cancer cell lines, based with a $P$ value $<0.05$ and $|\log 2(\mathrm{FC})|$ value $>0.50$. Then the relationship between the above genes and erlotinib resistance was explored.
Reagents and antibodies. Erlotinib was purchased from Shanghai Anpu Experiment Technology Co., dissolved in DMSO and maintained at $-20^{\circ} \mathrm{C}$. Antibodies against GAPDH (cat. no. 60004-1-lg), BAX (cat. no. 50599-2-lg), Bcl-2 (cat. no. 12789-1-AP), P21 (cat. no. 60214-1-lg), CDK4 (cat. no. 66950-1-lg), CDK6 (cat. no. 66278-1-lg), Cyclin D1 (cat. no. 60186-1-lg), E-cadherin (cat. no. 60335-1-lg), N-cadherin (cat. no. 66219-1-lg), GBP1 (cat. no. 15303-1-AP) and $\beta$-actin (cat. no. 60008-1-lg) were purchased from ProteinTech Group, Inc. and diluted at 1:500-1,000. Antibodies against cleaved PARP1 (cat. no. ab32561), Twist (cat. no. ab187008) and vimentin (cat. no. ab92547) were purchased from Abcam and a PGK1 (cat. no. sc-130335) antibody was purchased from Santa Cruz Biotechnology, Inc. All antibodies were diluted $1: 500$ or $1: 1,000$.

Establishment of cell lines. The human NSCLC cell lines PC9 (EGFR del19) and HCC827 (EGFR del19) were purchased from the American Type Culture Collection (ATCCP and cultured in RPMI-1640 (Gibco; Thermo Fisher Scientific, Inc.) medium containing $10 \%$ or $20 \%$ fetal bovine serum (FBS; Gibco; Thermo Fisher Scientific, Inc.). Erlotinib-resistant cell lines (PC9ER and HCC827ER) were generated via exposure to gradually increasing doses of erlotinib and were maintained in the presence of erlotinib after 6 months. In addition, the T790M mutation was not detected in the PC9ER and HCC827ER cells using a T790M test kit (HGN-eg02; Wuhan Haijili Biological Technology Co., China; Fig. S1A).

shRNA construction and transfection. Firstly, pCDH-CMVMCS-EF1-copGFP-Puro (4 $\mu \mathrm{g})$, pLP1 $(3 \mu \mathrm{g})$, pLP2 $(2 \mu \mathrm{g})$, and pLP-VSVG $(3 \mu \mathrm{g})$ were cotransfected into 293T cells, which were purchased from Addgene Co., and the virus titer was $0.98 \times 10^{8} \mathrm{TU} / \mathrm{ml}$. After PC9ER and HCC827ER cells reached $70 \%$ confluence $\left(3 \times 10^{6}\right.$ cells), lentiviral particles of GBP1 were used to transduce cells at a multiplicity of infection (MOI) of 50 in the presence of polybrene $(2 \mu \mathrm{g} / \mathrm{ml}$; Sigma-Aldrich; Merck KGaA). Afterwards, shRNA-transduced cells were screened with puromycin $(0.5 \mathrm{~g} / \mathrm{ml}$; Sigma-Aldrich; Merck KGaA) for $72 \mathrm{~h}$. Finally, the derived cell clones were successfully cultivated. In overexpression lentivirus, pGC-FU-3FLAG-SV40-puromycin-library (6 $\mu \mathrm{g})$, pHelper1.0 $(3 \mu \mathrm{g})$ and pHelper2.0 $(2 \mu \mathrm{g})$ were cotransfected into 293T cells, which were purchased from Genechem Co.; the virus titer was $1.5 \mathrm{E}+9 \mathrm{TU} / \mathrm{ml}$. After collection by centrifugation for $1 \mathrm{~h}$ at 30,000 rpm, overexpression lentiviruses were transduced into erlotinib-resistant cells with 20 MOI and used in subsequent experiments. The inserted sequences of two shRNAs and overexpression lentivirus are listed in Fig. S1E.

$R T-q P C R$. Total RNA was extracted from tumor cells with TRIzol (Invitrogen; Thermo Fisher Scientific, Inc.). The RNA concentration was detected by measuring the absorbance at $260 / 280 \mathrm{~nm}$. Then, cDNA was synthesized with a reverse transcription reagent kit (Invitrogen; Thermo Fisher Scientific, Inc.) according to the manufacturer's protocol. qPCR was performed with SYBR Green PCR Mater Mix (Takara Bio, Inc.). Finally, PCR was performed, under the following conditions: $10 \mathrm{~min}$ at $95^{\circ} \mathrm{C}$, followed by 40 cycles of $10 \mathrm{sec}$ at $94^{\circ} \mathrm{C}$, 
$30 \mathrm{sec}$ at $60^{\circ} \mathrm{C}$ and $30 \mathrm{sec}$ at $72^{\circ} \mathrm{C}$. The results were analyzed with the $2^{-\Delta \Delta C q}$ method (17). The sequences of primers for GBP1, GAPDH and E-cadherin are presented in Fig. S1B.

Drug sensitivity tests. Cell viability was measured by CCK-8 assays (Beyotime Institute of Biotechnology). Erlotinib-sensitive and erlotinib-resistant cells were placed into 96-well microplates, and erlotinib was added after $24 \mathrm{~h}$. After 48 h, CCK-8 reagent was added to each well. After incubation with CCK- 8 reagent at $37^{\circ} \mathrm{C}$ for $10 \mathrm{~min}$, the absorbance values in the microplate were detected by using a microplate reader. Three independent experiments were carried out.

Western blot (WB) analysis. After cells were collected in RIPA buffer, the protein was analyzed with a Pierce BCA protein assay (cat. no. 23227; Thermo Fisher Scientific, Inc.), and transferred onto polyvinylidene difluoride membranes (Bio-Rad Laboratories, Inc.). After blocking, the membranes were probed with primary antibodies overnight at $4^{\circ} \mathrm{C}$. After washing, the membranes were incubated with goat anti-rabbit immunoglobulin G (IgG) (cat. no. A0208; Beyotime Institute of Biotechnology) or goat anti-mouse IgG (cat. no. 7074 or 7076; Cell Signaling Technology, Inc.) for $1 \mathrm{~h}$ at $37^{\circ} \mathrm{C}$. Finally, an enhanced chemiluminescence detection reagent (Pierce; Thermo Fisher Scientific, Inc.) was applied to visualize the protein bands. Antibodies against GAPDH and $\beta$-actin served as controls. In Table SIA-E, the optimal density value of all WB bands are displayed, in which all comparisons between the two groups were statistically significant.

Apoptosis and cell cycle detection. After centrifugation for $5 \mathrm{~min}$ at $1000 \mathrm{rpm}$ and resuspension, $2 \times 10^{5}$ cells were stained with Annexin-V FITC and propidium iodide (PI) in the dark. Then, stained cells were resuspended in $400 \mu \mathrm{l}$ of binding buffer, and the samples were analyzed with a BD FACSVerse flow cytometer (BD Biosciences). Finally, the data were analyzed by FlowJo software v7.6.5 (Treestar Co.). In the cell cycle experiment, $1 \times 10^{6}$ cells were used and resuspended in ice-cold phosphate-buffered saline (PBS). Cold 100\% ethanol was slowly added to each tube. Then, the cells were incubated at $4^{\circ} \mathrm{C}$ overnight. After centrifugation for $5 \mathrm{~min}$ at $800 \mathrm{rpm}$ and washing, the cells were stained with $5 \mu \mathrm{l}$ of FITC-conjugated Annexin-V and $5 \mu \mathrm{l}$ of PI for $1 \mathrm{~h}$ and were analyzed. The data were processed by MODFIT software (Verity Software House).

Mouse xenograft study. Athymic nude mice (4 weeks old, female, $\mathrm{n}=32,4$ mice per group) were purchased from Southern Medical University Experimental Animal Research Center and housed under specific pathogen-free conditions; $5 \times 10^{6}$ PC9-overexpressing cells were subcutaneously injected into the flanks of nude mice on day 1 of the experiment. When the tumors reached $450 \mathrm{~mm}^{3}$ on day 7, erlotinib was intragastrically administered to 4 groups of mice once a day for 2 weeks. Tumor volume (Length $\mathrm{x} \mathrm{Width}^{2} / 2$ ) and body weight were measured and recorded. When the largest tumor approached $816 \mathrm{~mm}^{3}$ in the PC9-NC+PBS group on day 40 after resistant cell inoculation, all mice were euthanized by cervical dislocation. Next, the tumors were resected and analyzed by immunohistochemistry (IHC). None of the animals were found dead. According to humane spirit, the maximum volume of mice was limited to $4000 \mathrm{~mm}^{3}$. These studies were conducted in compliance with the guidelines from the Animal Care and Use Committee of Southern Medical University.

$I H C$. Tumor tissues were immediately fixed in $4 \%$ paraformaldehyde (Wuhan Boster Biological Technology, Ltd.) and embedded in paraffin. Samples were carefully sectioned, serially deparaffinized, rehydrated, and subjected to antigen retrieval. Monoclonal primary antibodies were incubated with samples for $1 \mathrm{~h}$ at $37^{\circ} \mathrm{C}$. Subsequently, after incubation with a suitable HRP-labeled secondary antibody for $30 \mathrm{~min}$, sections were stained with diaminobenzidine (OriGene Technologies, Inc.). Finally, sections were dehydrated and mounted. Meanwhile, the immunostained tumor cells were scored according to the following evaluation $(0,=$ negative; 1 , weak staining; 2 , moderate; 3 , strong), and the results are showed in Table SIIA and B, in which the comparison between the two groups were significant at $\mathrm{P}<0.01$.

Immunofluorescence and confocal microscopy. A total of $5 \times 10^{5}$ PC9ER or $2 \times 10^{5}$ HCC827ER cells were fixed with cold methanol for 10 min, permeabilized in $0.2 \%$ Triton X-100 for $15 \mathrm{~min}$ and blocked with sheep serum for $30 \mathrm{~min}$. After incubation with primary antibodies at $4^{\circ} \mathrm{C}$ overnight, cells were incubated with secondary antibodies for $1 \mathrm{~h}$ at $37^{\circ} \mathrm{C}$, and stained with 4',6-diamino-2-phenyl-indole (DAPI; cat. no. C1005; Beyotime Institute of Biotechnology). Images were captured on a confocal microscope with a x63 objective (TCS SP8; Leica) and analyzed by Leica application suite software (Leica Microsystems).

Coimmunoprecipitation (CoIP). For the CoIP experiments, $3 \times 10^{6}$ PC9ER cells were lysed in ice-cold Pierce lysis buffer (Thermo Fisher Scientific, Inc.) for $10 \mathrm{~min}$, followed by centrifugation at $13,000 \mathrm{x} \mathrm{g}$ for $10 \mathrm{~min}$. Then, cell lysates were incubated with antibody-crosslinked magnetic beads (Beyotime Institute of Biotechnology) for $1 \mathrm{~h}$. Normal IgG served as the control according to the instruction manual. Beads were washed with lysis buffer, and protein complexes were eluted in the next step. The input and CoIP samples were detected by WB analysis with primary antibodies against a variety of proteins.

Statistical analysis. We used a two-tailed Student's t-test or one-way ANOVA with GraphPad Prism 7 software (v8; GraphPad Software, Inc.) for statistical analysis and performed least significance difference (LSD) to obtain P-value for the one-way ANOVA. Statistical significance is indicated by $\mathrm{P}$-value with symbols as defined in the legends $\left({ }^{*} \mathrm{P}<0.05\right.$, ${ }^{* *} \mathrm{P}<0.01$ and $\left.{ }^{* * *} \mathrm{P}<0.001\right)$. Based on the median follow-up in months, the results of survival analysis were displayed using Kaplan-Meier survival curves by the log rank test and $\mathrm{P}<0.05$ indicated statistical significance. In addition, we utilized univariable and multivariate Cox regression to identify the variables affecting overall survival (OS) and clinical features in lung adenocarcinoma (LUAD). Image $\mathbf{J}$ software v1.8.0 (National Institutes of Health, Bethesda, MD, USA) was used to analyze gray value of the WB bands and the average optimal density of IHC. 

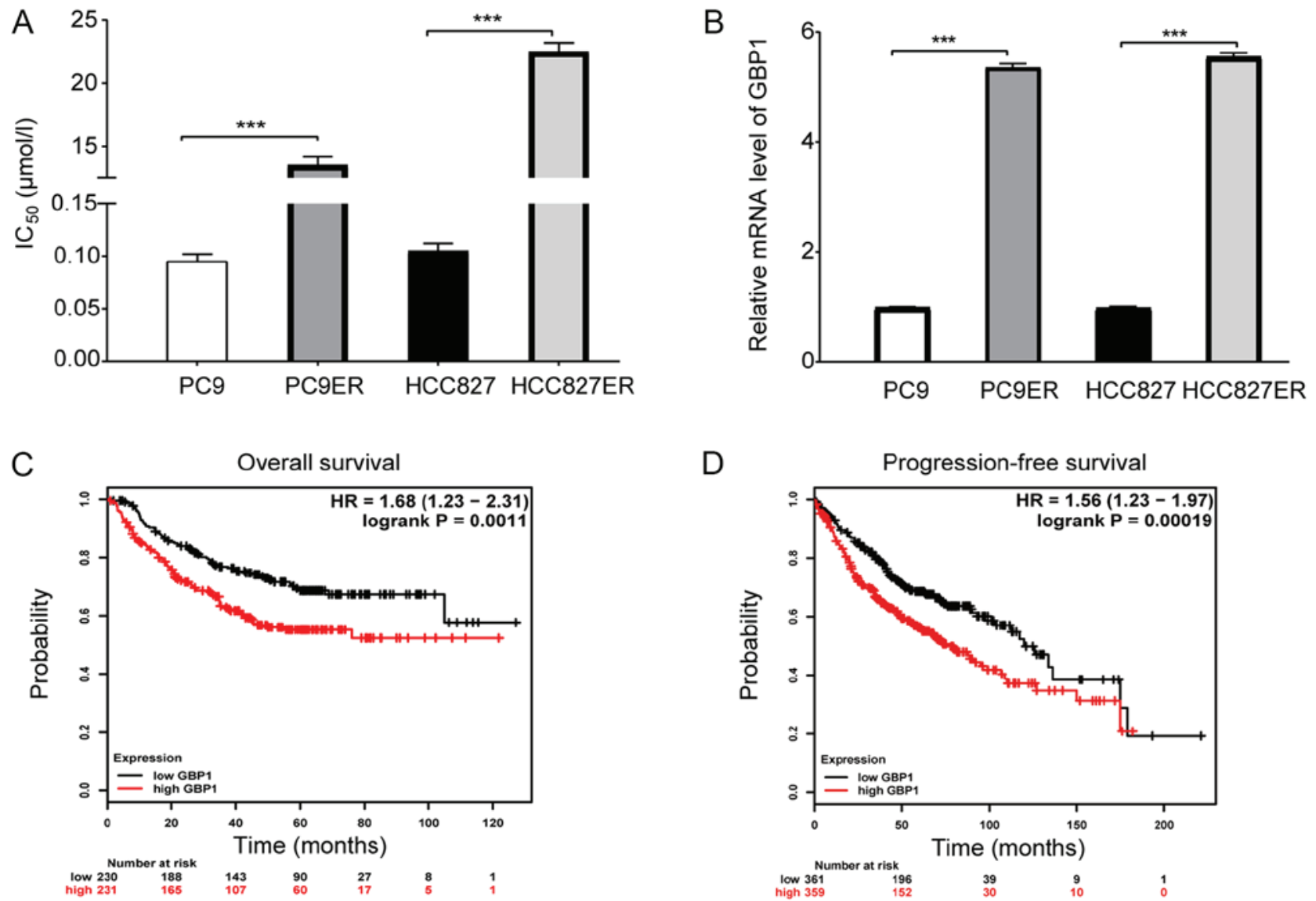

$\mathrm{D}$
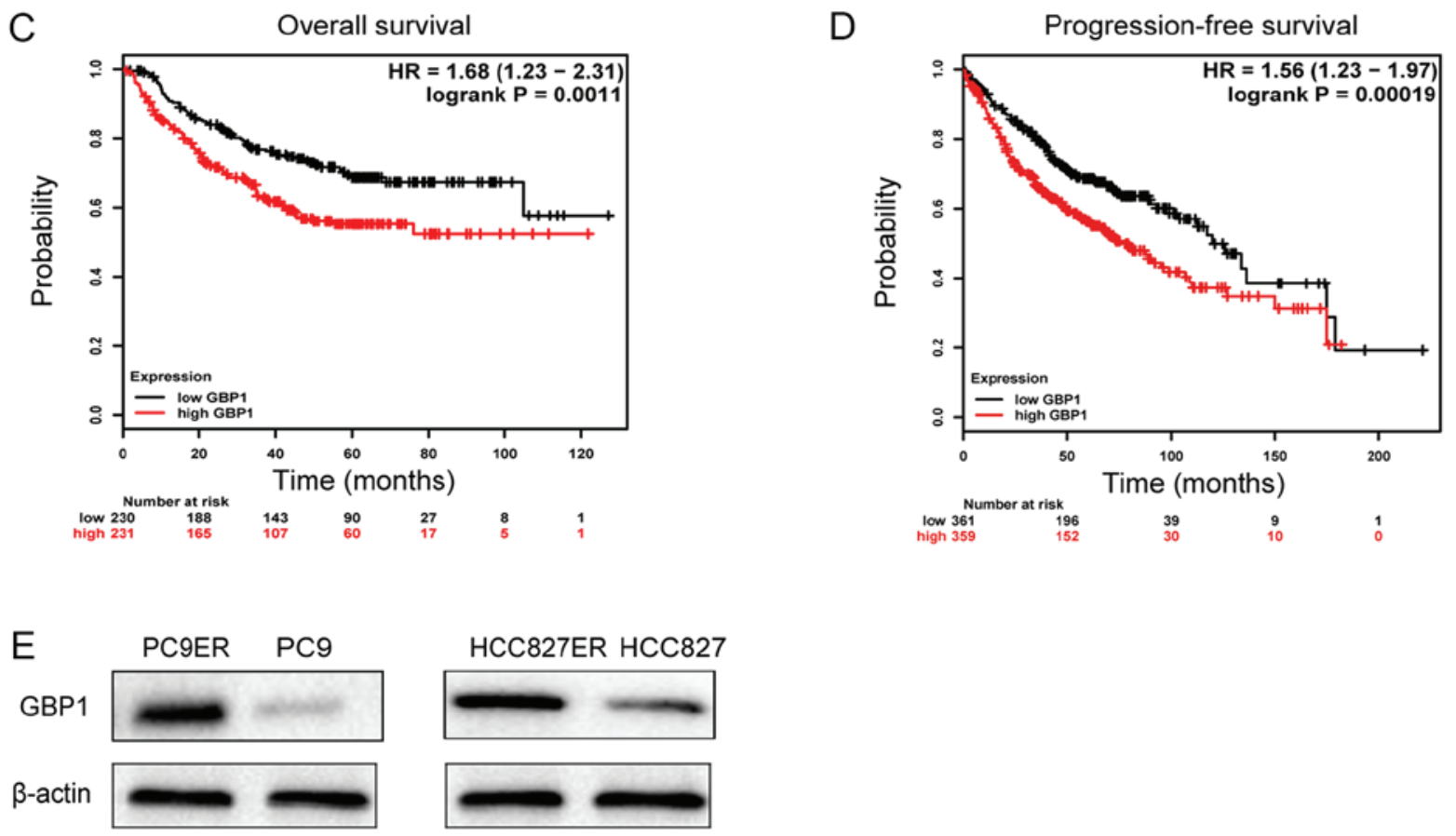

Figure 1. GBP1 expression is increased in PC9ER and HCC827ER cells. (A) Changes in $\mathrm{IC}_{50}$ were detected in erlotinib-sensitive (PC9 and HCC827) and -resistant (PC9ER and HCC827ER) cells by CCK-8 assay. (B) Expression of GBP1 mRNA was assessed in erlotinib-sensitive and -resistant cells. (C) OS and (D) PFS are shown between high GBP1 and low GBP1 groups in patients with lung adenocarcinoma. (E) Changes in the protein level of GBP1 was determined in erlotinib-sensitive and -resistant cells. ${ }^{* * * *} \mathrm{P}<0.001$. GBP1, guanylate-binding protein-1; $\mathrm{IC}_{50}$, half maximal inhibitory concentration; OS, overall survival; PFS, progression-free survival.

\section{Results}

GBP1 is upregulated in erlotinib-resistant cells. First, we screened different genes from datasets in the GEO database. Then, to investigate whether these genes were related to erlotinib resistance, we established erlotinib-resistant PC9 and HCC827 cell lines (PC9ER and HCC827ER) through stepwise induction. The half maximal inhibitory concentration $\left(\mathrm{IC}_{50}\right)$ value of erlotinib was significantly higher in the erlotinib-resistant cells than that noted in the corresponding erlotinib-sensitive cells as shown in Fig. 1A. Next, we analyzed the mRNA levels of these genes in erlotinib-resistant and erlotinib-sensitive cells. Finally, GBP1 (Fig. 1B) and TGM2 (Fig. S1C) were selected due to the significant differences in their expression. However, there have been many studies on TGM2 and drug resistance, thus we chose to focus on $G B P 1$ in this study. Subsequently, we explored the relationship of these genes with prognosis through Kaplan-Meier Plotter analysis. As shown in Fig. 1C and D, patients were divided into a high-level group and a low-level GBP1 group according to the median GBP1 expression value (10.4684), and overall survival (OS) and progression-free survival (PF) were shorter in the high-level group than in the low-level group among patients with lung adenocarcinoma. Fig. 1C includes 230 lung adenocarcinoma patients with low GBP1 expression and 231 patients with high GBP1 expression from The Cancer Genome Atlas (TCGA) database. A total of 620 lung adenocarcinoma patients with PF time data were analyzed, as shown in Fig. 1D. Moreover, we found that high GBP1 expression was an independent prognostic factor of OS in LUAD (Table I), and related to tumor stage in 234 patients with LUAD from the Oncomine database (https://www.oncomine .org/resource/login.html; Fig. S1D). In addition, GBP1 protein levels were differentially expressed in the erlotinib-sensitive and erlotinib-resistant cells (Fig. 1E). Overall, GBP1 was found to be related to erlotinib resistance and worthy of further study. 
Table I. Univariate and multivariate analyses of overall survival.

Overall survival

\begin{tabular}{|c|c|c|c|c|}
\hline \multirow[b]{3}{*}{ Features } & \multirow{2}{*}{\multicolumn{2}{|c|}{ Univariate analysis }} & \multirow{2}{*}{\multicolumn{2}{|c|}{ Multivariate analysis }} \\
\hline & & & & \\
\hline & Hazard ratio $(95 \% \mathrm{CI})$ & P-value & Hazard ratio $(95 \% \mathrm{CI})$ & P-value \\
\hline \multicolumn{5}{|l|}{ Age (years) } \\
\hline$<65$ & 1 (reference) & & 1 (reference) & \\
\hline$\geq 65$ & $0.773(0.505-1.181)$ & 0.233 & $0.613(0.373-1.009)$ & 0.054 \\
\hline \multicolumn{5}{|l|}{ Sex } \\
\hline Male & 1 (reference) & & 1 (reference) & \\
\hline Female & $1.137(0.746-1.732)$ & 0.552 & $0.892(0.561-1.418)$ & 0.629 \\
\hline \multicolumn{5}{|l|}{ Pathologic stage } \\
\hline Stage I & 1 (reference) & & 1 (reference) & \\
\hline Stage II & $0.276(0.126-0.604)$ & $0.001^{\mathrm{a}}$ & $0.469(0.191-1.149)$ & 0.098 \\
\hline Stage III & $0.570(0.255-1.274)$ & 0.171 & $0.686(0.274-1.714)$ & 0.420 \\
\hline Stage IV & $1.160(0.518-2.598)$ & 0.719 & $1.941(0.740-5.092)$ & 0.178 \\
\hline \multicolumn{5}{|c|}{ Anatomic neoplasm subdivision } \\
\hline L-upper & 1 (reference) & & 1 (reference) & \\
\hline L-lower & $1.001(0.482-2.077)$ & 0.998 & $1.021(0.468-2.228)$ & 0.958 \\
\hline R-upper & $0.756(0.439-1.302)$ & 0.313 & $0.607(0.340-1.082)$ & 0.090 \\
\hline R-lower & $1.390(0.792-2.438)$ & 0.251 & $0.786(0.413-1.496)$ & 0.463 \\
\hline R-middle & $1.213(0.286-5.143)$ & 0.793 & $0.247(0.050-1.233)$ & 0.088 \\
\hline \multicolumn{5}{|c|}{ Primary therapy outcome } \\
\hline Progressive disease & 1 (reference) & & 1 (reference) & \\
\hline Partial response & $2.331(1.120-4.851)$ & $0.024^{\mathrm{a}}$ & $2.703(1.253-5.830)$ & $0.011^{\mathrm{a}}$ \\
\hline Complete response & $0.000(0.000-1.528 \mathrm{E}+239)$ & $<0.001^{\mathrm{a}}$ & $0.000(0.000-1.693 \mathrm{E}+238)$ & 0.965 \\
\hline Stable disease & $0.477(0.231-0.984)$ & $0.045^{\mathrm{a}}$ & $1.689(0.760-3.754)$ & 0.198 \\
\hline \multicolumn{5}{|l|}{ Radiation therapy } \\
\hline Yes & 1 (reference) & & 1 (reference) & \\
\hline No & $3.136(2.001-4.915)$ & $<0.001^{\mathrm{a}}$ & $1.535(0.854-2.760)$ & 0.152 \\
\hline \multicolumn{5}{|l|}{ Neoplasm cancer status } \\
\hline Tumor-free & 1 (reference) & & 1 (reference) & \\
\hline With tumor & $0.149(0.090-0.246)$ & $<0.001^{\mathrm{a}}$ & $0.160(0.087-0.293)$ & $<0.001^{\circ}$ \\
\hline \multicolumn{5}{|l|}{ GBP1 expression level } \\
\hline Low expression & 1 (reference) & & 1 (reference) & \\
\hline High expression & $1.810(1.179-2.777)$ & $0.007^{\mathrm{a}}$ & $2.136(1.303-3.501)$ & $0.003^{3}$ \\
\hline
\end{tabular}

${ }^{a} \mathrm{P}<0.05$, statistically significant. GBP1, guanylate-binding protein-1; CI, confidence interval.

$G B P 1$ regulates the sensitivity of erlotinib-resistant cells in vitro. To confirm the role of GBP1, gain- and loss-of-function experiments were performed. After lentivirus transfection, shGBP1-transfected PC9ER cells had significantly lower GBP1 mRNA and protein levels than these levels in the control cells (Fig. 2A and B). In addition, Fig. $2 \mathrm{C}$ shows that the $\mathrm{IC}_{50}$ value for erlotinib was several times higher in the PC9ER-NC cells than this value in the shGBP1-transfected PC9ER cells. Similar to the results in PC9ER cells, the results in the HCC827ER cells showed the same trend (Fig. 2A-C). In the overexpression assay, resistance to erlotinib was higher in the GBP1-overexpressing PC9 (PC9-GBP1) and HCC827 (HCC827-GBP1) cells than in the corresponding negative control-transfected PC9 (PC9-NC) and HCC827 (HCC827-NC) cells (Fig. 2D-F), demonstrating that GBP1 overexpression contributed to erlotinib resistance as indicated by a notable change in the $\mathrm{IC}_{50}$ of erlotinib. Based on these data, PC9ER and HCC827ER cells transfected with shRNA became sensitive to erlotinib, but the overexpression of GBP1 in PC9 and HCC827 cells conferred erlotinib resistance, indicating that GBP1 regulates erlotinib resistance in vitro.

Knockdown of GBP1 induces cell apoptosis in erlotinib-resistant cells. After cells had been treated for $48 \mathrm{~h}$ with erlotinib at a concentration close to its $\mathrm{IC}_{50}$, flow cytometry analysis revealed that the proportion of late apoptotic cells was 13.1, 20.1 and $20.5 \%$ in the PC9ER-NC, PC9ER-shGBP1-1 and 

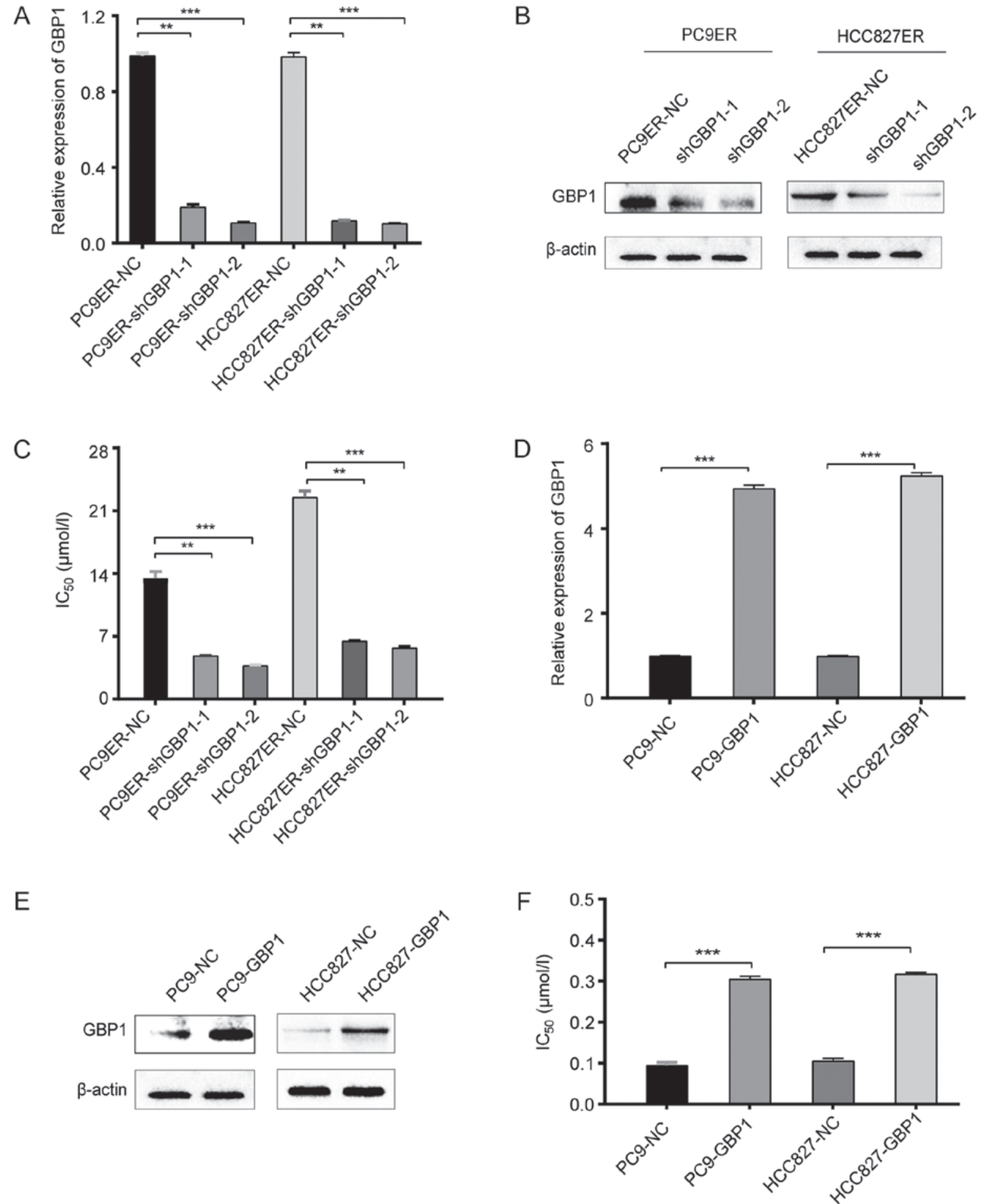

Figure 2. GBP1 induces erlotinib resistance in vitro. (A) GBP1 mRNA level was determined following the knock down of GBP1 (shGBP1-1 and -2) in erlotinib-resistant cells (PC9ER and HCC827ER). (B) GBP1 protein level was determined following knock down of GBP1 in erlotinib-resistant cells. (C) IC s0 $_{5}$ values were determined after downregulation of GBP1 in erlotinib-resistant cells. (D) GBP1 mRNA level was determined following GBP1 upregulation (-GBP1) in erlotinib-sensitive cells (PC9 and HCC827). (E) GBP1 protein level was determined after overexpression of GBP1 in erlotinib-sensitive cells. (F) $\mathrm{IC}_{50}$ values were determined following upregulation of GBP1 in erlotinib-sensitive cells. ${ }^{* *} \mathrm{P}<0.01,{ }^{* * * *} \mathrm{P}<0.001$ when compared to the relevant negative control (NC) group. GBP1, guanylate-binding protein-1; $\mathrm{IC}_{50}$, half maximal inhibitory concentration.

PC9ER-shGBP1-2 cells, respectively (Fig. 3A). Unlike HCC827ER-NC cells, the ratio of late apoptotic cells among HCC827ER-shGBP1-1 and HCC827ER-shGBP1-2 cells was 34.5 and $36.9 \%$ respectively (Fig. 3B). Moreover, the overexpression of GBP1 in erlotinib-sensitive cells (PC9-GBP1 and HCC827-GBP1) decreased the percentage of late apoptotic cells compared with that in the NC cells (Fig. 3C and D). Using WB analyses, we determined the levels of the apoptotic markers, Bax, Bcl-2 and cleaved PARP1. When GBP1 was knocked down in erlotinib-resistant cells, cleaved PARP1 and Bax protein levels were increased, and the change in Bcl-2 protein levels showed opposite trends (Fig. 3E). After GBP1 
A

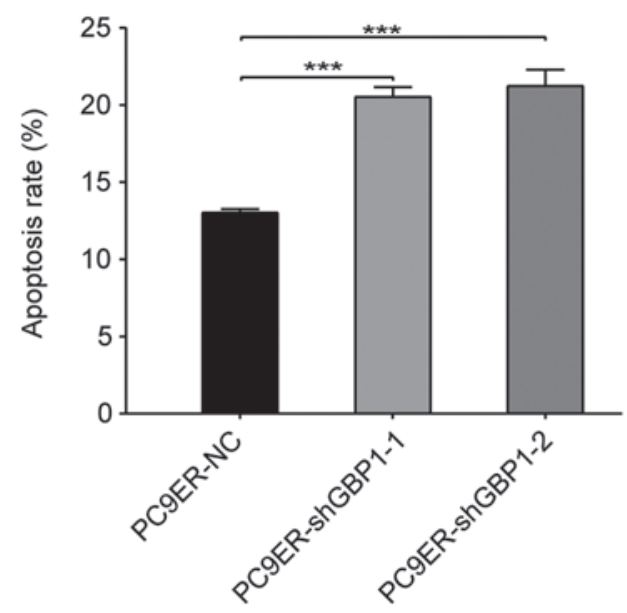

C

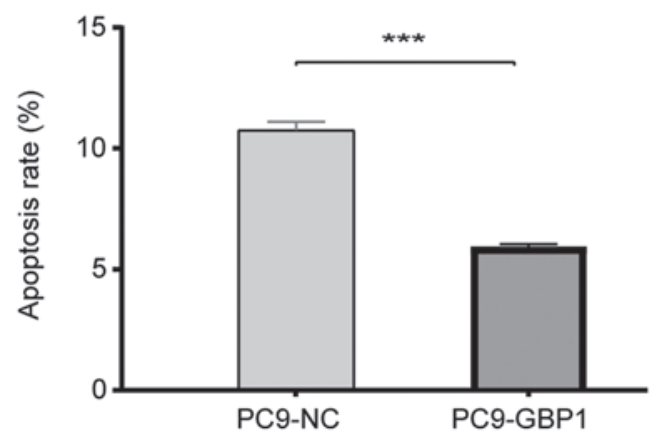

B

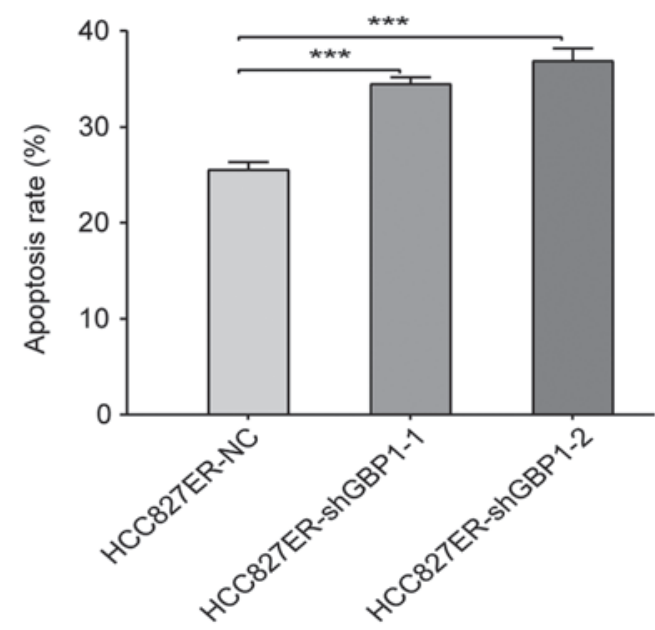

D

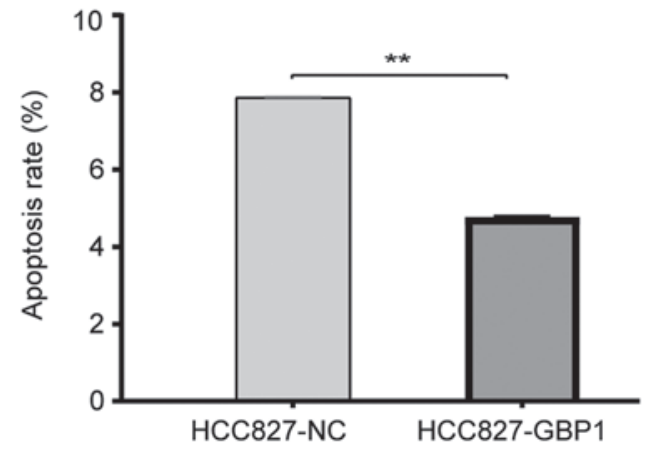

$E$
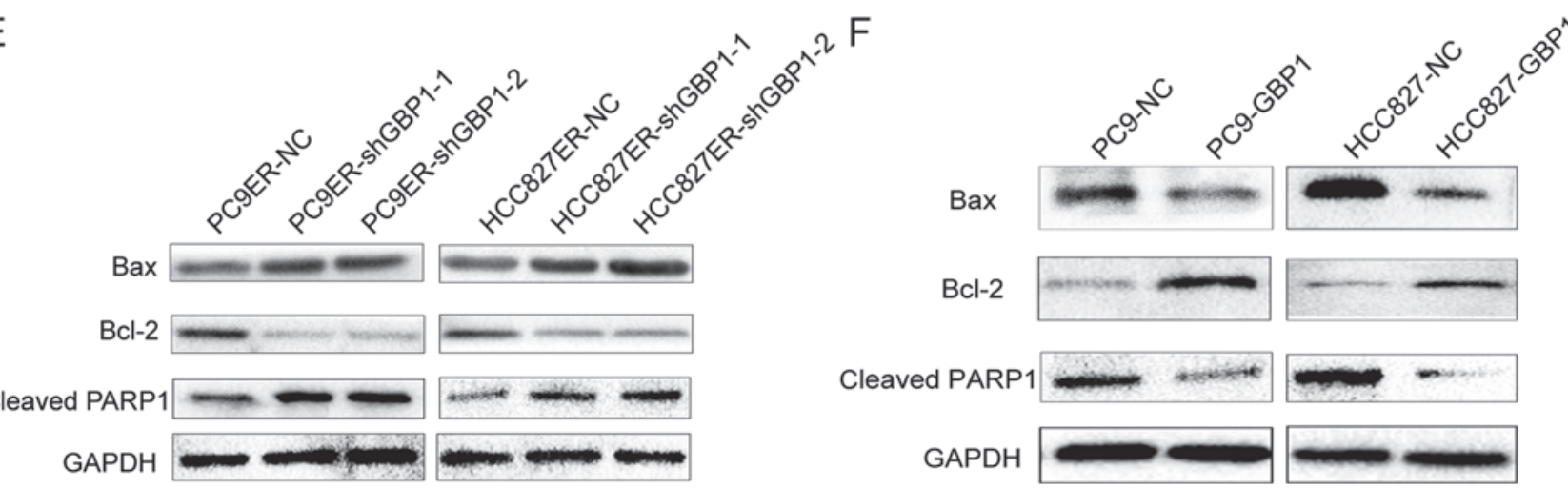

Figure 3. GBP1 regulates apoptosis in NSCLC cells. (A and B) The percentage of late apoptotic cells was analyzed by flow cytometry following knockdown of GBP1 (shGBP1-1 and -2) in erlotinib-resistant cells (PC9ER and HCC827ER). (C and D) The percentage of late apoptotic cells was analyzed by flow cytometry following upregulation of GBP1 (-GBP1) in erlotinib-sensitive cells (PC9 and HCC827). (E and F) Bax, Bcl-2, cleaved PARP1 protein levels were determined by western blot analysis. ${ }^{* *} \mathrm{P}<0.01,{ }^{* * *} \mathrm{P}<0.001$ when compared to the relevant negative control (NC) group. GBP1, guanylate-binding protein-1; NSCLC, non-small cell lung cancer; Bax, bcl-2-like protein 4; Bcl-2, B-cell lymphoma 2; PARP1, poly(ADP-ribose) polymerase 1.

overexpression, the opposite effects were observed (Fig. 3F). All of the above results showed that GBP1 is involved in erlotinib resistance via the apoptotic pathway.

GBP1 induces a G1 phase transition. Then, we explored the role of GBP1 in the cell cycle. By performing flow cytometry analysis, we discovered that the proportions of shGBP1-1 and shGBP1-2-transfected PC9ER cells in the G1 phase were 73.1 and $85.1 \%$, respectively (Fig. 4A), while the proportion of PC9-GBP1 cells in the G1 phase was $57.0 \%$, which significantly differed from the respective control cells (Fig. 4C). There was also a significant difference in the proportion of
shGBP1-transfected HCC827ER cells and control cells in the G1 phase (Fig. 4B), and the proportion of HCC827-GBP1 cells and control cells in the G1 phase (Fig. 4D). P21 is an important regulator of the G1 cell cycle checkpoint (18), and cyclin D1, CDK4 and CDK6 regulate the G0/G1 transition in the cell cycle (19). The P21 protein level was elevated when GBP1 was downregulated in PC9ER or HCC827ER cells (Fig. 4E), and the other three markers exhibited an opposite effect. But when GBP1 was overexpressed, the P21 protein level was decreased (Fig. 4F). All of these results regarding cell cycle proteins showed that GBP1 regulates the cell cycle and leads to a G1 phase transition. 
A

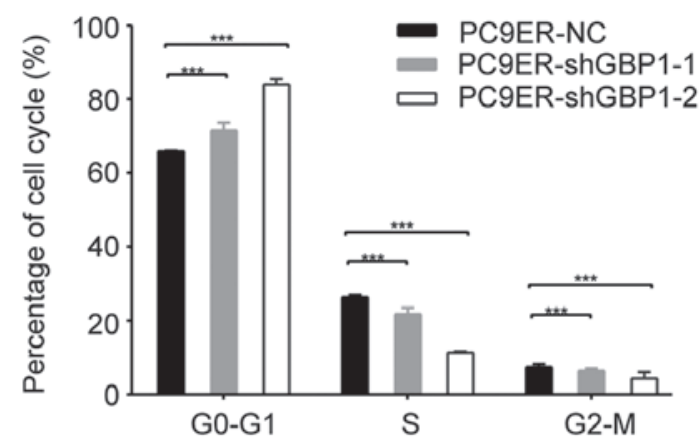

C

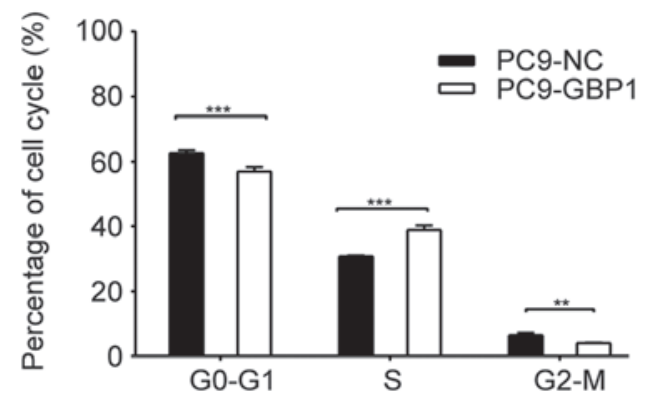

$\mathrm{B}$

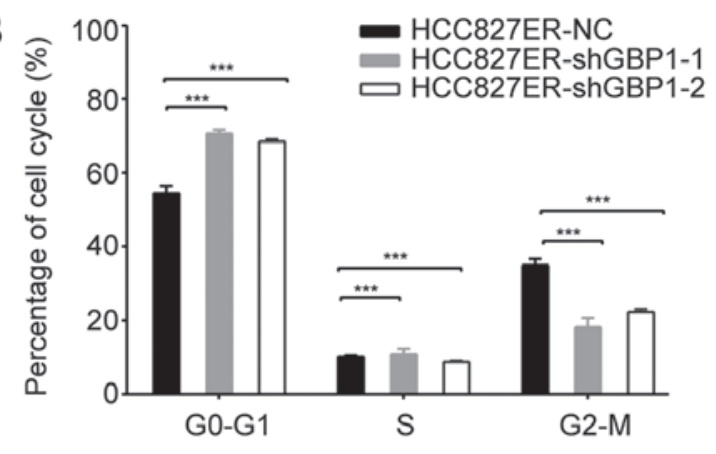

D

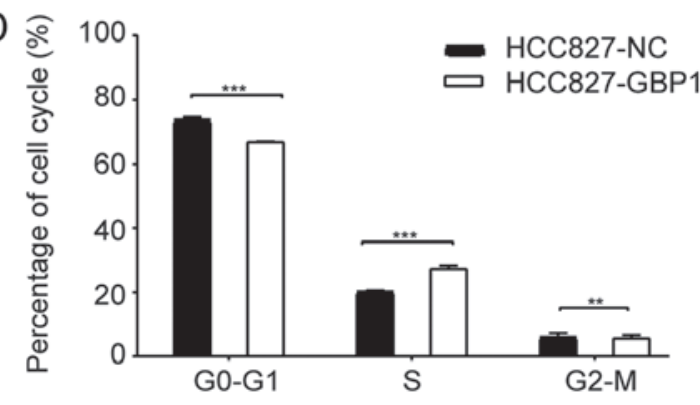

E

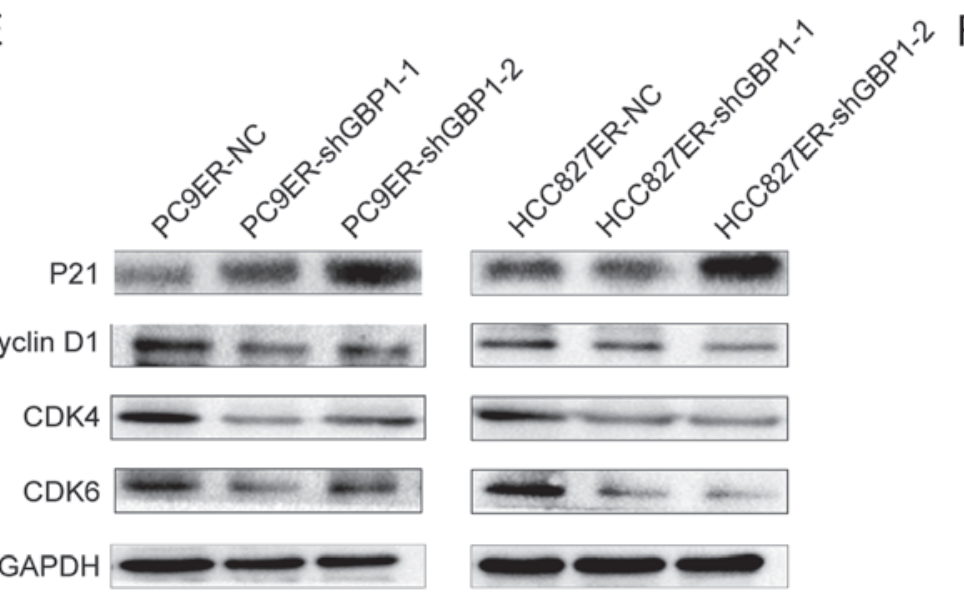

$\mathrm{F}$

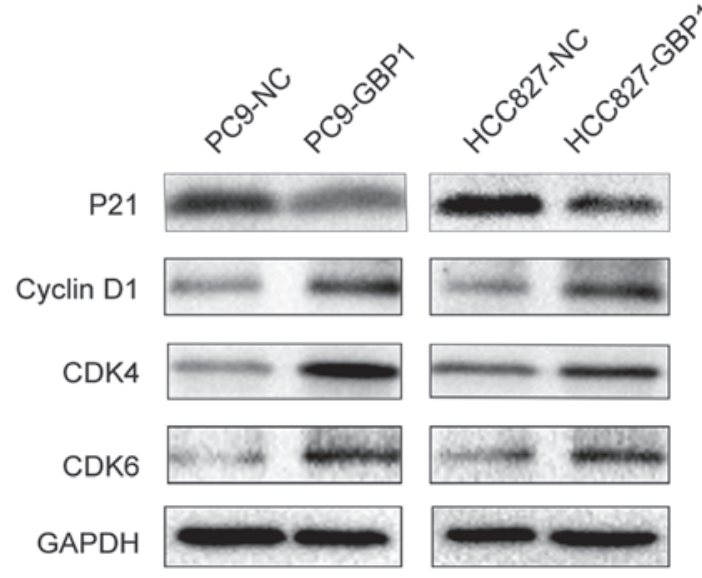

Figure 4. GBP1 regulates the cell cycle by flow cytometry. (A and B) The cell cycle ratio was analyzed by flow cytometry following knockdown of GBP1 (shGBP1-1 and -2) in erlotinib-resistant cells (PC9ER and HCC827ER). (C and D) Cell cycle ratio was analyzed by flow cytometry following the upregulation of GBP1 (-GBP1) in erlotinib-sensitive cells (PC9 and HCC827). (E and F) P21, cyclin D1, CDK4 and CDK6 protein levels were determined by western blot analysis. ${ }^{* *} \mathrm{P}<0.01,{ }^{* * * *} \mathrm{P}<0.001$ when compared to the relevant negative control (NC) group. CDK, cyclin-dependent kinase; GBP1, guanylate-binding protein-1.

GBP1 is associated with erlotinib resistance in mouse xenografts. To further verify the role of GBP1 in erlotinib resistance, we performed subcutaneous tumor formation experiments in nude mice. The tumor size in mice injected with PC9-GBP1 cells and treated with erlotinib was significantly larger than that in mice injected with PC9-NC and treated with erlotinib (Fig. 5A and B), which showed that the overexpression of GBP1 made the tumors resistant to erlotinib. Overall, the results of animal experiments showed that erlotinib sensitivity was regulated by either GBP1 knockout or overexpression, which once again verified the relationship between GBP1 and erlotinib resistance. In addition, the expression of apoptotic proteins was examined using IHC (Fig. 5C and D), and the results were consistent with those of the in vitro experiments.
Compared to PC9-NC + erlotinib, the expression of cleaved PARP1 in PC9-GBP1 + erlotinib was decreased (Fig. 5D). Overall, the in vivo studies showed that GBP1 can regulate erlotinib resistance.

GBP1 contributes to erlotinib resistance by interacting with $P G K 1$. To explore GBP1-interacting proteins, we performed mass spectrometry, which can predict hundreds of possible interacting proteins (Fig. 6A). After analyzing the results, we selected PGK1, myosin light chain 9 (MYL9), annexin A2 (ANXA2) and aldolase A (ALDOA) as our research objects based on a total score $>200$ with $>5$ qualitative peptides. As PGK1 may be involved in erlotinib resistance (20), we chose PGK1 for further analysis. As shown in Fig. 6B, CoIP experiments showed 
A

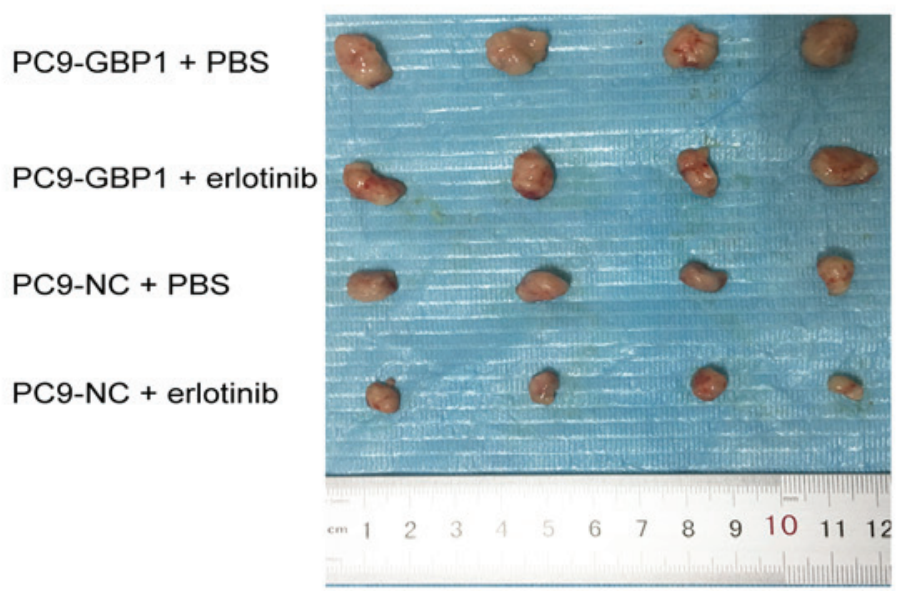

C

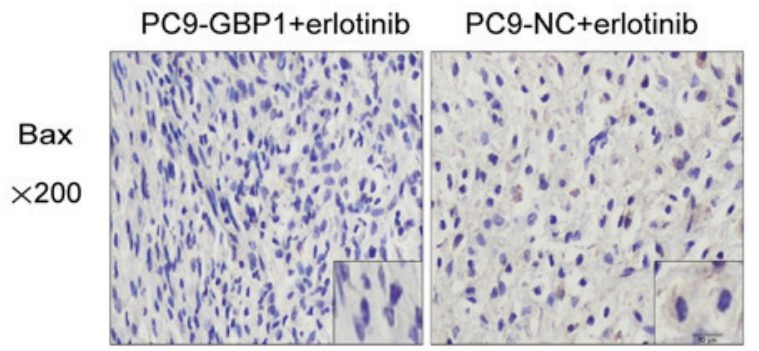

B

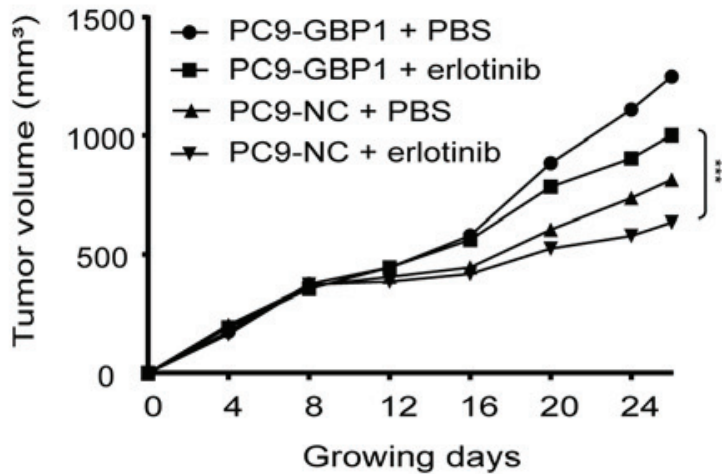

PC9-GBP1+erlotinib PC9-NC+erlotinib

$\mathrm{Bcl}-2$

$\times 200$
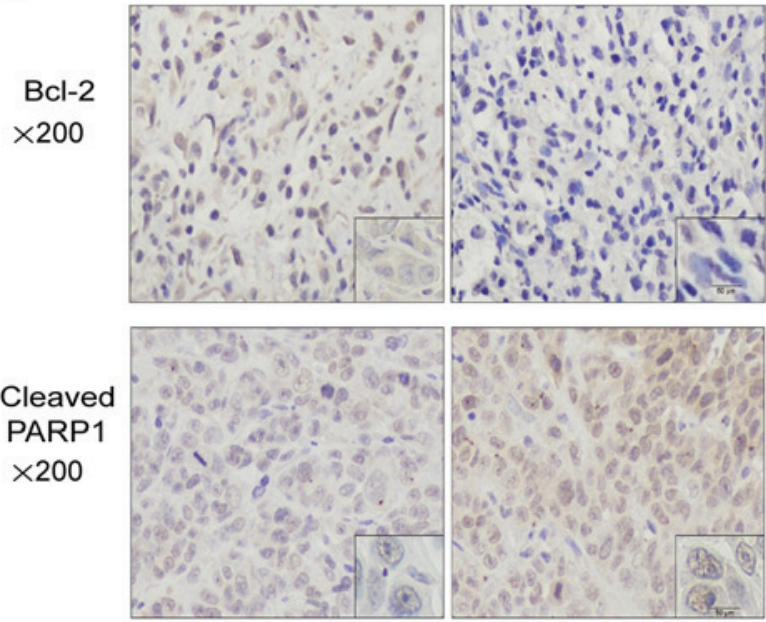

Figure 5. GBP1 is involved in erlotinib resistance in vivo. (A) Images of tumors in each group (PC9-GBP1+PBS, PC9-GBP1+erlotinib, PC9-NC+PBS and PC9-NC+erlotinib) are shown. (B) Curve of the tumor volume in the nude mice were determined in the different groups. (C) Expression of Bax by immunohistochemistry (IHC) in captured mouse tumors is shown in the PC9ER-NC+erlotinib and PC9-GBP1+erlotinib group (D) Expression of Bcl-2 and cleaved PARP1 by immunohistochemistry (IHC) in captured mouse tumors is shown in the PC9ER-NC+erlotinib and PC9-GBP1+erlotinib group. ${ }^{* * *} \mathrm{P}<0.001$ when PC9-NC+erlotinib vs. PC9-GBP1+erlotinib group. Magnification, x200. GBP1, guanylate-binding protein-1; Bax, bcl-2-like protein 4; Bcl-2, B-cell lymphoma 2; PARP1, poly(ADP-ribose) polymerase 1.

that PGK1 interacted with the GBP1 protein. Next, the results of immunofluorescence staining showed the colocalization of GBP1 and PGK1 in the two resistant cell lines (Fig. 6C). Analysis of the cBio Cancer Genomics Portal, showed that the mRNA expression level of PGK1 was positively correlated with that of GBP1 $(\mathrm{P}<0.05)$ and the Spearman correlation coefficient between GBP1 and PGK1 expression in lung adenocarcinoma was 0.31 (Fig. 6D). Taken together, these results showed that GBP1 interacts with PGK1. To determine whether PGK1 affects erlotinib resistance in NSCLC, we used CCK-8 assays and found that PGK1 downregulation reduced the $\mathrm{IC}_{50}$ of erlotinib (Fig. 6E). Finally, the relationship between PGK1 and prognosis was examined through Kaplan-Meier Plotter analysis; high PGK1 expression predicted poor OS and PF in lung adenocarcinoma (Fig. 6F and G). In conclusion, GBP1 regulates erlotinib resistance via PGK1.

EMT mediated by PGK1 plays an important role in GBP1-regulated erlotinib resistance. To determine which downstream pathways are involved in resistance, we examined the literature and found that PGK1 can interact with HIF-2 to promote EMT in breast cancer (20). In addition, EMT is closely related to EGFR-TKI resistance in many types of tumors, so we explored the relationship between GBP1, PGK1 and EMT with erlotinib resistance. WB data showed that the knockdown of GBP1 induced a marked increase in E-cadherin levels and decreased $\mathrm{N}$-cadherin and vimentin levels. However increased PGK1 expression reversed changes in the protein levels of E-cadherin, N-cadherin and vimentin (Fig. 7A). PGK1 downregulation after GBP1 overexpression reversed the effect of GBP1 on EMT-related proteins (Fig. 7B). Additionally, Twist, as a key factor in EMT, was decreased at the protein level when PGK1 was inhibited using siRNA (Fig. 7C), further supporting that PGK1 regulated EMT. In Fig. 7D, the mRNA level of E-cadherin in resistant cells was decreased when compared to that in sensitive cells. In addition, we further analyzed the change in the $\mathrm{IC}_{50}$ caused by E-cadherin downregulation in erlotinib-sensitive cells and found that reduced E-cadherin 


\begin{tabular}{ll|l|l|l}
\hline A & Protein name & Score & Coverage & Peptide \\
\cline { 2 - 4 } MYL9 & 770 & 37.8 & 6 \\
\hline ANXA2 & 604 & 34.5 & 10 \\
\hline ALDOA & 259 & 25.8 & 9 \\
\hline PGK1 & 229 & 22.1 & 6 \\
\hline OPA1 & 219 & 7 & 5 \\
\hline DDX17 & 188 & 11.1 & 7 \\
\hline BAF & 160 & 29.2 & 2 \\
\hline YBOX & 155 & 13.7 & 3 \\
\hline RS4X & 150 & 20.2 & 6 \\
\hline TRM6 & 149 & 8.9 & 4
\end{tabular}

B

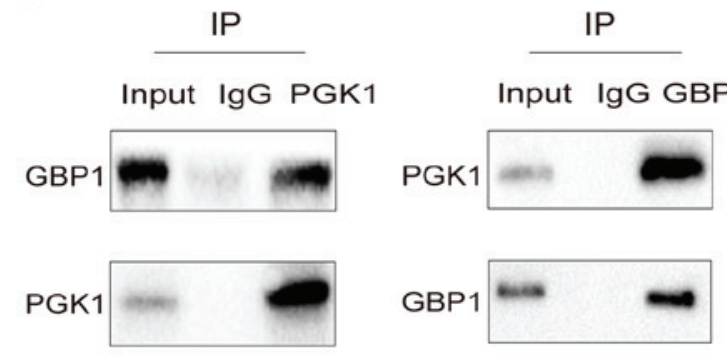

C
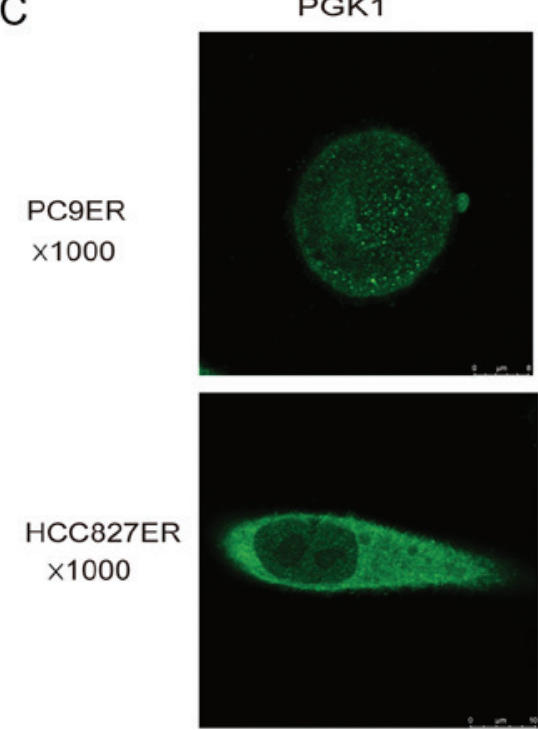

D

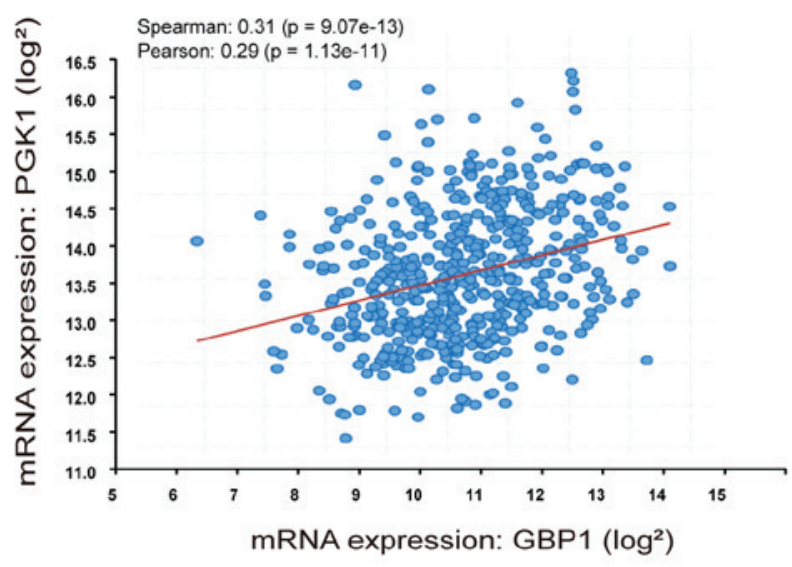

GBP1
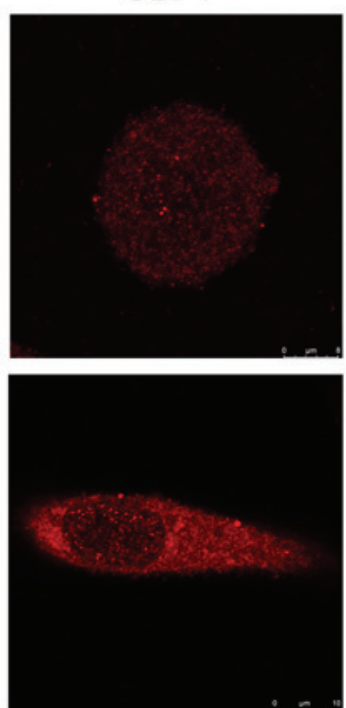

E
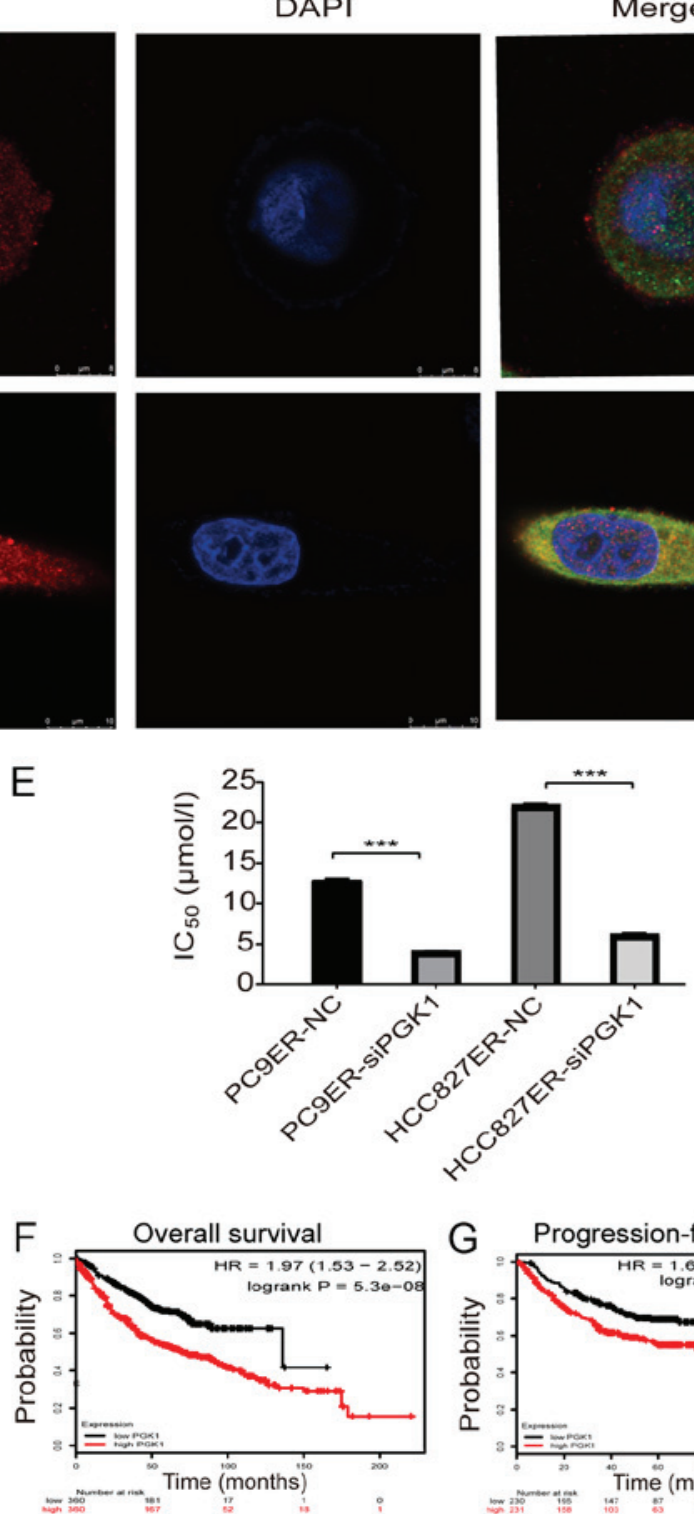
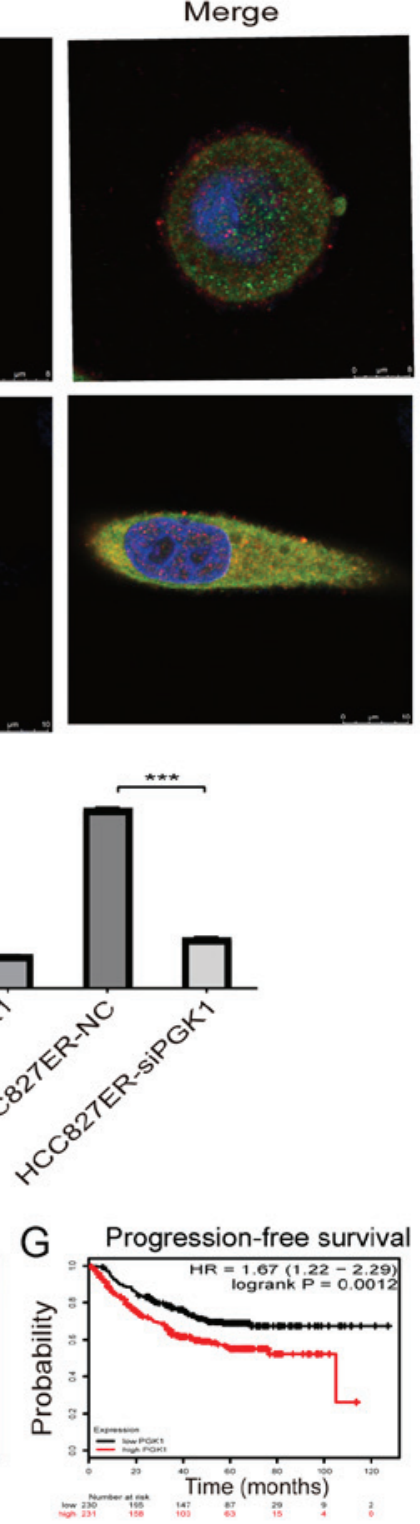

Figure 6. GBP1 and PGK1 interact to regulate erlotinib resistance. (A) Mass spectrum analysis displayed possible interactive proteins with GBP1. (B) The interaction of GBP1 and PGK1 was detected by coimmunoprecipitation (CoIP) experiment. (C) The colocalization of GBP1 and PGK1 are presented by immunofluorescence staining. (D) The correlation between GBP1 and PGK1 was demonstrated by cBioportal database. (E) IC $\mathrm{C}_{50}$ values were determined after downregulation of PGK1 (-siPGK1) in erlotinib-resistant (PC9ER and HCC827ER) cells. ${ }^{* * *} \mathrm{P}<0.001$, compared with the relevant negative control (NC) group. (F) OS and (G) PFS are shown between high PGK1 and low PGK1 groups in the patients with lung adenocarcinoma. GBP1, guanylate-binding protein-1; PGK1, phosphoglycerate kinase $1 ; \mathrm{IC}_{50}$, half maximal inhibitory concentration; OS, overall survival; PFS, progression-free survival.

led to erlotinib resistance (Fig. 7E). These results indicated that EMT participates in the regulation of erlotinib resistance. Finally, as shown in Fig. 7F and G, we found that the results of histochemical staining for PGK1 and EMT proteins were consistent with those of WB. Moreover, the immunostaining score of PGK1 and EMT proteins are displayed in Table SIIA. 
A

$\mathrm{N}$-cadherin (PC9ER)

Vimentin (PC9ER)

GAPDH

E-cadherin (HCC827ER)

$\mathrm{N}$-cadherin (HCC827ER)

Vimentin (HCC827ER)

GAPDH
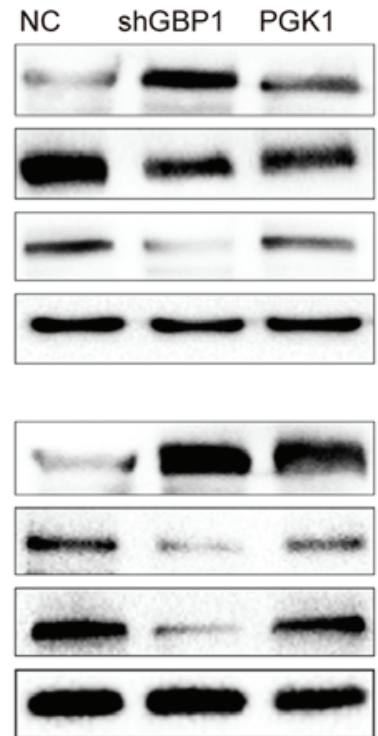

B

E-cadherin (PC9)

$\mathrm{N}$-cadherin (PC9)

Vimentin (PC9)

GAPDH

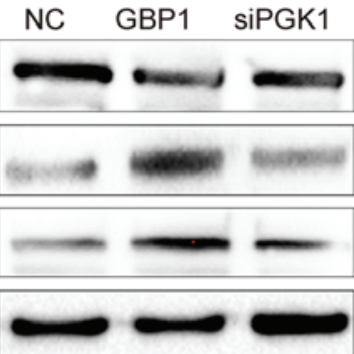

E-cadherin ( $\mathrm{HCC} 827)$

$\mathrm{N}$-cadherin $(\mathrm{HCC} 827)$

Vimentin (HCC827)

GAPDH

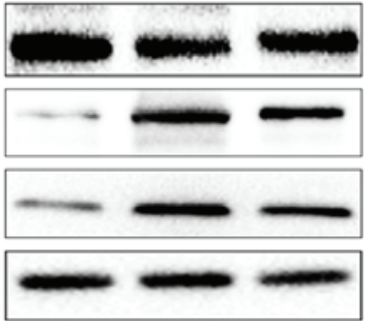

C
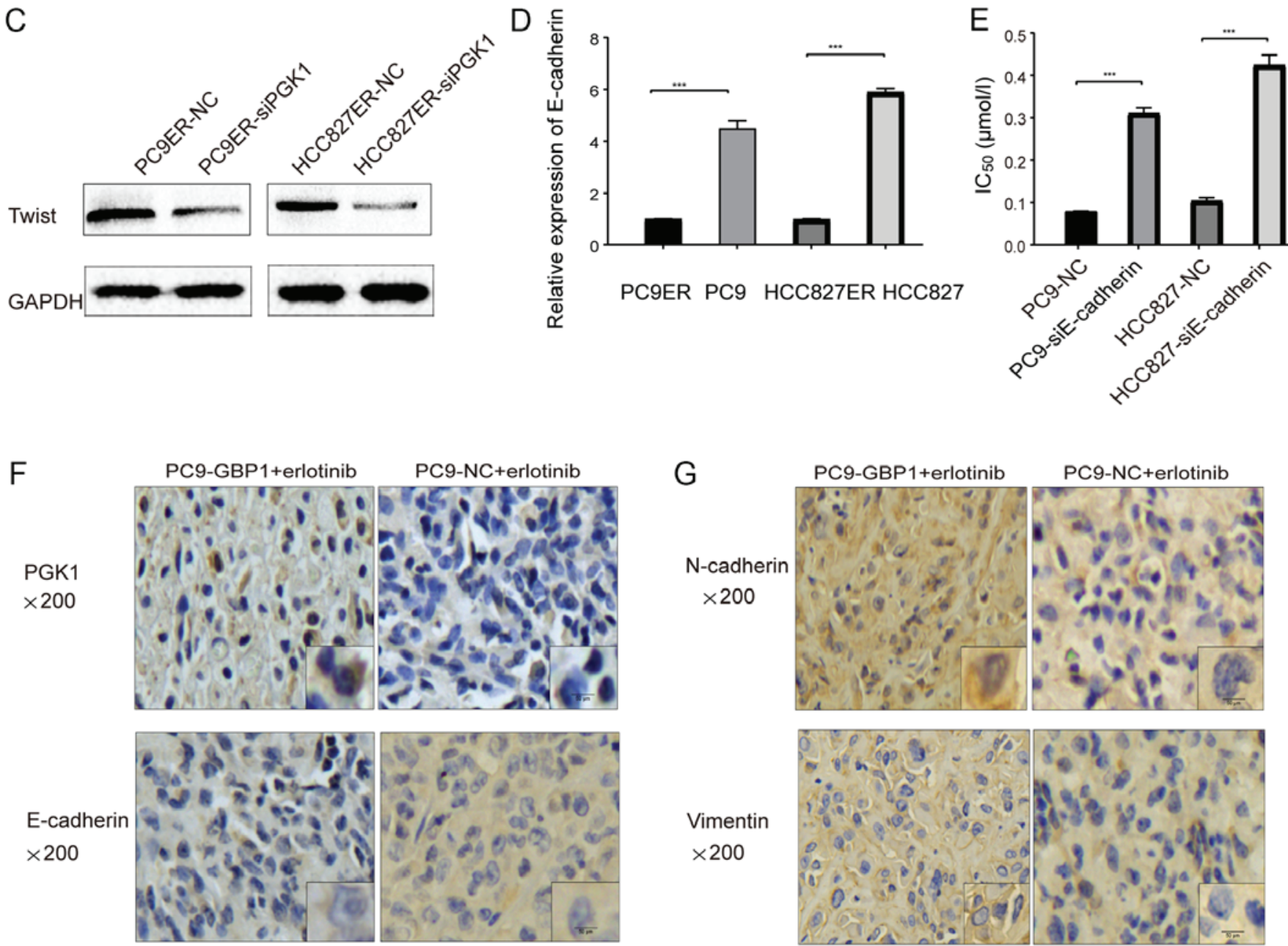

Figure 7. EMT pathway mediated by PGK1 plays an important role in GBP1-modulated erlotinib resistance. (A and B) PGK1 restores the effect of GBP1 on the levels of EMT-related proteins, E-cadherin, N-cadherin and vimentin, in both erlotinib-resistant (PC9ER and HCC827ER) (A) and sensitive (PC9 and HCC827) cells (B). (C) Change in Twist protein was displayed after downregulation of PGK1 (-siPGK1) in erlotinib-resistant (PC9ER and HCC827ER) cells compared with the NC groups. (D) E-cadherin mRNA levels were determined in sensitive (PC9 and HCC827) and resistant (PC9ER and HCC827ER) cells. (E) $\mathrm{IC}_{50}$ values were determined in erlotinib-sensitive cells following E-cadherin downregulation (-siE-cadherin). ${ }^{* * * *} \mathrm{P}<0.001$, when compared with the relevant negative control (NC) group. (F and G) Expression of PGK1, E-cadherin, N-cadherin and vimentin by immunohistochemistry (IHC) are showed in animal tumor samples. ETM, epithelial-mesenchymal transition; GBP1, guanylate-binding protein-1; PGK1, phosphoglycerate kinase 1; $\mathrm{IC}_{50}$, half maximal inhibitory concentration. 
Changes in protein levels of E-cadherin, $\mathrm{N}$-cadherin and vimentin indicated again that GBP1 could activate EMT. The above experiments showed that GBP1 induces erlotinib resistance via the EMT pathway, which is activated by PGK1 in NSCLC. In the future, further analysis of the relationship between EMT and erlotinib resistance through EMT inhibitors or agonists must be performed.

\section{Discussion}

By interrupting the epidermal growth factor receptor (EGFR) signaling pathway, erlotinib is highly successful as an initial treatment for non-small cell lung cancer (NSCLC), but resistance limits the clinical utility of erlotinib. A detailed understanding of its mechanism is critical for patients to benefit from an optimal therapeutic response. There are many reports concerning epidermal growth factor receptor tyrosine kinase inhibitor (EGFR-TKI) resistance. Kobayashi et al, reported that the T790M mutation is a common mechanism of EGFR-TKI resistance (21). Oser et al found that a histological transition from NSCLC to small cell lung cancer (SCLC) is a mechanism of EGFR-TKI resistance (22). However, many mechanisms of EGFR-TKI resistance remain unclear.

In the present study, we found that guanylate-binding protein-1 (GBP1) expression was increased in erlotinib-resistant cells. Previous research has shown that GBP1 expression is high in lung adenocarcinoma (8) and colorectal cancer (23). Wadi et al reported that elevated GBP1 was correlated to a shorter progression-free time in ovarian cancer (24). In addition, studies have reported that GBP1 overexpression is associated with paclitaxel resistance (25). However, it remains unknown whether GBP1 is involved in the regulation of EGFR-TKI resistance. Our experiments showed that the downregulation of GBP1 in erlotinib-resistant cells increased erlotinib sensitivity, while GBP1 overexpression in erlotinib-sensitive cells promoted erlotinib resistance. Moreover, our study revealed that GBP1 regulates apoptosis and the cell cycle. In vivo models also indicated that GBP1 is associated with erlotinib resistance. Furthermore, survival analysis showed that high GBP1 levels predict poor prognosis. Overall, these results demonstrate that GBP1 induces erlotinib resistance in NSCLC.

To explore the mechanism by which GBP1 regulates erlotinib resistance, we performed mass spectrometry to identify GBP1 interacting proteins. By CoIP experiments, we identified phosphoglycerate kinase 1 (PGK1) as a GBP1-interacting protein. Several studies reported an association between PGK1 and chemotherapeutic resistance. Zhou et al observed that PGK1 induced cisplatin resistance in endometrioid adenocarcinoma (26). In a recent study, the blockade of PGK1 was shown to overcome chemotherapeutic resistance in gastric cancer (27), and elevated PGK1 was found to promote paclitaxel resistance in breast cancer (28). However, a relationship between PGK1 and erlotinib resistance has not been reported. In the present study, we found that PGK1 regulates erlotinib resistance through CCK-8 assays. Therefore, inhibiting the GBP1-PGK1 axis may be an effective way to overcome erlotinib resistance.

Several studies have suggested that PGK1 facilitates cell invasion viaEMT in breast cancer (29). Epithelial-mesenchymal transition (EMT) is a biological process that is involved in cell proliferation invasion, migration and chemotherapy resistance in NSCLC. Specifically, EMT induces erlotinib resistance in NSCLC (30), and Lou et al also reported that EMT generates cellular erlotinib resistance via the STAT3 pathway in NSCLC (31). In addition, the EMT status was found to influence EGFR-TKI resistance via the Wnt or Notch pathway in EGFR-mutant NSCLC cancer (32). Weng et al also showed that EMT is a common mechanism of EGFR-TKI resistance (33). Therefore, we further evaluated the importance of the EMT signaling pathway in mediating erlotinib resistance via PGK1 and discovered that GBP1 and PGK1 strongly depend on the EMT pathway to regulate erlotinib resistance. Additionally, the downregulation of E-cadherin in erlotinib-sensitive cells led to a change in erlotinib sensitivity. All the above results showed that EMT is a key step in erlotinib resistance and that the activation of the EMT pathway by PGK1 promotes erlotinib resistance mediated by GBP1 in NSCLC.

In summary, the present study found that GBP1 is a critical modulator of erlotinib resistance and is expected to provide basic experimental evidence for 'targeting GBP1 to reduce EGFR-TKI resistance'.

\section{Acknowledgements}

The authors thank the staff involved in the present study.

\section{Funding}

This research was supported by the National Natural Science Foundation of China (grant nos. 81772457 and 81871859), The Province Medical Scientific Research Foundation of Guangdong (grant no. A2018246), and The Province Natural Science Foundation of Guangdong (grant no. 2018A030310471).

\section{Availability of data and materials}

All data are included in this article and its supplementary information files.

\section{Authors' contributions}

LC collected the data and wrote the manuscript. LG reviewed the manuscript. TW analyzed the results. JZ contributed to the conception and design of the research. All authors were involved in some aspect of conducting the experiments. All authors read and approved the manuscript and agree to be accountable for all aspects of the research in ensuring that the accuracy or integrity of any part of the work are appropriately investigated and resolved.

\section{Ethics approval and consent to participate}

The present study was approved by the Ethics Committee of Southern Medical University (Guangzhou, China).

\section{Patient consent for publication}

Not applicable. 


\section{Competing interests}

The authors declare that there are no competing interests.

\section{References}

1. Siegel R, Naishadham D and Jemal A: Cancer statistics, 2013. CA Cancer J Clin 63: 11-30, 2013.

2. Jänne PA, Wang X, Socinski MA, Crawford J, Stinchcombe TE, Gu L, Capelletti M, Edelman MJ, Villalona-Calero MA, Kratzke R, et al: Randomized phase II trial of erlotinib alone or with carboplatin and paclitaxel in patients who were never or light former smokers with advanced lung adenocarcinoma: CALGB 30406 trial. J Clin Oncol 30: 2063-2069, 2012.

3. Yu HA, Arcila ME, Rekhtman N, Sima CS, Zakowski MF, Pao W, Kris MG, Miller VA, Ladanyi M and Riely GJ: Analysis of tumor specimens at the time of acquired resistance to EGFR-TKI therapy in 155 patients with EGFR-mutant lung cancers. Clin Cancer Res 19: 2240-2247, 2013.

4. Suda K, Rivard CJ, Mitsudomi T and Hirsch FR: Overcoming resistance to EGFR tyrosine kinase inhibitors in lung cancer, focusing on non-T790M mechanisms. Expert Rev Anticancer Ther 17: 779-786, 2017.

5. Westover D, Zugazagoitia J, Cho BC, Lovly CM and Paz-Ares L: Mechanisms of acquired resistance to first- and second-generation EGFR tyrosine kinase inhibitors. Ann Oncol 29 (suppl_1): i10-i19, 2018.

6. Britzen-Laurent N, Lipnik K, Ocker M, Naschberger E, Schellerer VS, Croner RS, Vieth M, Waldner M, Steinberg P, Hohenadl C, et al: GBP-1 acts as a tumor suppressor in colorecta cancer cells. Carcinogenesis 34: 153-162, 2013.

7. Yu CJ, Chang KP, Chang YJ, Hsu CW, Liang Y, Yu JS, Chi LM, Chang YS and Wu CC: Identification of guanylate-binding protein 1 as a potential oral cancer marker involved in cell invasion using omics-based analysis. J Proteome Res 10: 3778-3788, 2011.

8. Yamakita I, Mimae T, Tsutani Y, Miyata Y, Ito A and Okada M: Guanylate binding protein 1 (GBP-1) promotes cell motility and invasiveness of lung adenocarcinoma. Biochem Biophys Res Commun 518: 266-272, 2019.

9. Tipton AR, Nyabuto GO, Trendel JA, Mazur TM, Wilson JP, Wadi S, Justinger JS, Moore GL, Nguyen PT and Vestal DJ: Guanylate-binding Protein-1 protects ovarian cancer cell lines but not breast cancer cell lines from killing by paclitaxel. Biochem Biophys Res Commun 478: 1617-1623, 2016.

10. Ji X, Zhu H, Dai X, Xi Y, Sheng Y, Gao C, Liu H, Xue Y, Liu J, Shi J, et al: Overexpression of GBP1 predicts poor prognosis and promotes tumor growth in human glioblastoma multiforme. Cancer Biomark 25: 275-290, 2019.

11. Quintero M, Adamoski D, Reis LMD, Ascenção CFR, Oliveira KRS, Gonçalves KA, Dias MM, Carazzolle MF and Dias SMG: Guanylate-binding protein-1 is a potential new therapeutic target for triple-negative breast cancer. BMC Cancer 17: 727, 2017.

12. Yu T, Zhao Y, Hu Z, Li J, Chu D, Zhang J, Li Z, Chen B, Zhang X, Pan $\mathrm{H}$, et al: MetaLnc9 facilitates lung cancer metastasis via a PGK1-activated AKT/mTOR pathway. Cancer Res 77: 5782-5794, 2017

13. Niederst MJ, Sequist LV, Poirier JT, Mermel CH, Lockerman EL, Garcia AR, Katayama R, Costa C, Ross KN, Moran T, et al: $\mathrm{RB}$ loss in resistant EGFR mutant lung adenocarcinomas that transform to small-cell lung cancer. Nat Commun 6: 6377, 2015.

14. Zhang Z, Lee JC, Lin L, Olivas V, Au V, LaFramboise T, Abdel Rahman M, Wang X, Levine AD, Rho JK, et al: Activation of the AXL kinase causes resistance to EGFR-targeted therapy in lung cancer. Nat Genet 44: 852-860, 2012.

15. Nakata A, Yoshida R, Yamaguchi R, Yamauchi M, Tamada Y, Fujita A, Shimamura T, Imoto S, Higuchi T, Nomura M, et al: Elevated $\beta$-catenin pathway as a novel target for patients with resistance to EGF receptor targeting drugs. Sci Rep 5: 13076, 2015.
16. Smyth GK: Limma: linear models for microarray. In: Bioinformatics and Computational Biology Solutions Using $R$ and Bioconductor. Gentleman R, Carey VJ, Huber W, Irizarry RA and Dudoit S (eds). Springer, New York, NY, pp397-420, 2005.

17. Livak KJ and Schmittgen TD: Analysis of relative gene expression data using real-time quantitative PCR and the 2(-Delta Delta C(T)) Method. Methods 25: 402-408, 2001.

18. Xiong Y, Hannon GJ, Zhang H, Casso D, Kobayashi R and Beach D: p21 is a universal inhibitor of cyclin kinases. Nature 366: 701-704, 1993.

19. Massagué J: G1 cell-cycle control and cancer. Nature 432: 298-306, 2004

20. Yang H, Geng YH, Wang P, Zhou YT, Yang H, Huo YF, Zhang HQ, Li Y, He HY, Tian XX, et al: Extracellular ATP promotes breast cancer invasion and epithelial-mesenchymal transition via hypoxia-inducible factor $2 \alpha$ signaling. Cancer Sci 110: 2456-2470, 2019.

21. Kobayashi S, Boggon TJ, Dayaram T, Jänne PA, Kocher O, Meyerson M, Johnson BE, Eck MJ, Tenen DG and Halmos B: EGFR mutation and resistance of non-small-cell lung cancer to gefitinib. N Engl J Med 352: 786-792, 2005.

22. Oser MG, Niederst MJ, Sequist LV and Engelman JA: Transformation from non-small-cell lung cancer to small-cell lung cancer: Molecular drivers and cells of origin. Lancet Oncol 16: e165-e172, 2015.

23. Thomas H: Colorectal cancer: CRC endothelial regulation. Nat Rev Gastro Hepat 13: 682, 2016.

24. Wadi S, Tipton AR, Trendel JA, Khuder SA and Vestal DJ: hGBP-1 expression predicts shorter progression-free survival in ovarian cancers, while contributing to paclitaxel resistance. J Cancer Ther 7: 994-1007, 2016.

25. Duan Z, Foster R, Brakora KA, Yusuf RZ and Seiden MV: GBP1 overexpression is associated with a paclitaxel resistance phenotype. Cancer Chemother Pharmacol 57: 25-33, 2006.

26. Zhou JW, Tang JJ, Sun W and Wang H: PGK1 facilities cisplatin chemoresistance by triggering HSP90/ERK pathway mediated DNA repair and methylation in endometrial endometrioid adenocarcinoma. Mol Med 25: 11, 2019.

27. SchneiderCC,Archid R, Fischer N, BühlerS, Venturelli S, Berger A, Burkard M, Kirschniak A, Bachmann R, Königsrainer A, et al: Metabolic alteration--Overcoming therapy resistance in gastric cancer via PGK-1 inhibition in a combined therapy with standard chemotherapeutics. Int J Surg 22: 92-98, 2015.

28. Sun S, Liang X, Zhang X, Liu T, Shi Q, Song Y, Jiang Y, Wu H, Jiang Y, Lu X, et al: Phosphoglycerate kinase-1 is a predictor of poor survival and a novel prognostic biomarker of chemoresistance to paclitaxel treatment in breast cancer. Br J Cancer 112: 1332-1339, 2015

29. Fu D, He C, Wei J, Zhang Z, Luo Y, Tan H and Ren C: PGK1 is a potential survival biomarker and invasion promoter by regulating the HIF-1alpha-mediated epithelial-mesenchymal transition process in breast cancer. Cell Physiol Biochem 51: 2434-2444, 2018.

30. Byers LA, Diao L, Wang J, Saintigny P, Girard L, Peyton M, Shen L, Fan Y, Giri U, Tumula PK, et al: An epithelial-mesenchymal transition gene signature predicts resistance to EGFR and PI3K inhibitors and identifies Axl as a therapeutic target for overcoming EGFR inhibitor resistance. Clin Cancer Res 19: 279-290, 2013.

31. Lou W, Chen Y, Zhu KY, Deng H, Wu T and Wang J: Polyphyllin I overcomes EMT-associated resistance to erlotinib in lung cancer cells IL-6/STAT3 pathway inhibition. Biol Pharm Bull 40: 1306-1313, 2017.

32. Jakobsen KR, Demuth C, Sorensen BS and Nielsen AL: The role of epithelial to mesenchymal transition in resistance to epidermal growth factor receptor tyrosine kinase inhibitors in non-small cell lung cancer. Transl Lung Cancer Res 5: 172-182, 2016.

33. Weng CH, Chen LY, Lin YC, Shih JY, Lin YC, Tseng RY, Chiu AC, Yeh YH, Liu C, Lin YT, et al: Epithelial-mesenchymal transition (EMT) beyond EGFR mutations per se is a common mechanism for acquired resistance to EGFR TKI. Oncogene 38: 455-468, 2019. 